\title{
A central limit theorem for mixing random fields
}

\author{
Zsolt Karácsony
}




\title{
A CENTRAL LIMIT THEOREM FOR MIXING RANDOM FIELDS
}

\author{
ZSOLT KARÁCSONY
}

[Received: February 01, 2006]

\begin{abstract}
Detailed proofs of central limit theorems are given for mixing random fields. The results cover the case when the locations of the observations become dense in an increasing sequence of domains.

Mathematics Subject Classification: 60F05, 62M30

Keywords: central limit theorem, $\alpha$-mixing, random field, asymptotic normality of estimators, infill asymptotics, increasing domain asymptotic
\end{abstract}

\section{INTRODUCTION}

Ibragimov and Linnik [6] proved a central limit theorem (CLT) for stationary sequences satisfying certain $\alpha$-mixing conditions. Bolthausen [1] and Guyon [11] extended it to $\alpha$-mixing random fields. Fazekas [10] and Fazekas and Kukush [8] presented the so-called infill-increasing versions of Guyon's result for the bounded and the uniformly integrable cases, respectively. These papers do not contain the proofs of the theorems mentioned (a sketch of the proof can be found in [9]). The aim of our paper is to give proofs for the above mentioned theorems. The importance of the detailed proof is the following. It turns out that the original proof by Ibragimov and Linnik [6] and Guyon [11] can be applied if the random field satisfies a certain uniform integrability condition. Guyon [11] does not assume the uniform integrability but he does not describe the step from the bounded case to the general case. Therefore we do not know if his result is valid in the general case. We mention that Ibragimov and Linnik [6] assumed stationarity, so their proof is complete.

Our Theorem 1 contains the bounded case (it is a version of the result of [10]) while Theorem 2 is the general central limit theorem (it is contained in [7]). Theorems 3 and 4 are the $p$-dimensional extensions of Theorems 1 and 2, respectively.

There is a vast literature of mixing random processes. Bradley [2] gives a recent survey of mixing conditions. Recent advances in the central limit theorems for random sequences are considered by Merlevède, Peligrad and Utev in [13].

In [4] a general CLT is proved for stationary random fields. As is pointed out in [4], certain mixing conditions imply the assumptions of the general CLT. 
Note that our mixing conditions are not comparable with the ones in [4]. Moreover, in that paper stationary field and fixed designs are studied, while in our paper we do not assume stationarity and consider an infill-increasing setup.

\section{NOTATION AND PRELIMINARY REMARKS}

The following notation is used. $\mathbb{Z}$ is the set of all integers, $\mathbb{Z}^{d}$ is the set of $d$ dimensional lattice points where $d$ is a fixed positive integer. $\mathbb{R}$ is the real line, $\mathbb{R}^{d}$ is the $d$-dimensional space with the usual Euclidean norm $\|\boldsymbol{x}\|$. In $\mathbb{R}^{d}$ we shall also consider the distance corresponding to the maximum norm

$$
\varrho(\boldsymbol{x}, \boldsymbol{y})=\max _{1 \leq i \leq d}\left|x_{i}-y_{i}\right|
$$

where $\boldsymbol{x}=\left(x_{1}, \ldots, x_{d}\right), \boldsymbol{y}=\left(y_{1}, \ldots, y_{d}\right)$. The distance of two sets in $\mathbb{R}^{d}$ corresponding to the maximum norm is also denoted by $\varrho: \varrho(A, B)=\inf \{\varrho(\boldsymbol{x}, \boldsymbol{y}): \boldsymbol{x} \in$ $A, y \in B\}$.

For real valued sequences $\left\{a_{n}\right\}$ and $\left\{b_{n}\right\}, a_{n}=\mathrm{o}\left(b_{n}\right)$ (resp., $\left.a_{n}=\mathrm{O}\left(b_{n}\right)\right)$ means that the sequence $a_{n} / b_{n}$ converges to 0 (resp., is bounded). We shall denote different constants with the same letter $c$. I $\{A\}$ denotes the indicator function of the set $A$. $|\mathscr{D}|$ denotes the cardinality of the finite set $\mathscr{D}$.

We shall suppose the existence of an underlying probability space $(\Omega, \mathcal{F}, \mathbb{P})$. The $\sigma$-algebra generated by a set of events or by a set of random variables will be denoted by $\sigma\{\cdot\}$. The sign $\mathbb{E}$ stands for the expectation. The variance and covariance are denoted by $\operatorname{var}(\cdot)$ and $\operatorname{cov}(\cdot, \cdot)$, respectively. The $L_{p}$-norm of a random (vector) variable $\eta$ is defined as

$$
\|\eta\|_{p}=\left\{\mathbb{E}\|\eta\|^{p}\right\}^{1 / p}, 1 \leq p<\infty .
$$

The sign " $\Rightarrow$ " denotes the convergence in distribution. $\mathcal{N}(m, \Sigma)$ stands for the (vector) normal distribution with the mean (vector) $m$ and covariance (matrix) $\Sigma$.

The scheme of observations is the following. Let $T_{1}, T_{2}, \ldots$, and $T_{\infty}$ be domains in $\mathbb{R}^{d}$. Suppose that $T_{1} \subset T_{2} \subset T_{3} \subset \ldots, \bigcup_{i=1}^{\infty} T_{i}=T_{\infty}$. Assume that $T_{i}$ is compact for each $i, T_{\infty}$ is of infinite Lebesgue measure. Let $\left\{\varepsilon(\boldsymbol{x}), \boldsymbol{x} \in T_{\infty}\right\}$ be a random field. The $n$-th set of observations consists of values of the random field $\varepsilon(\boldsymbol{x})$ taken at points $\boldsymbol{x}_{\boldsymbol{k}} \in T_{n}$, where $\boldsymbol{k} \in \mathbb{D}_{n} \subset \mathbb{Z}^{d}$. The choice of points $\boldsymbol{x}_{\boldsymbol{k}}$ is the following. Divide $\mathbb{R}^{d}$ into hyperrectangles

$$
\Delta_{n}(\boldsymbol{k})=\prod_{j=1}^{d}\left(\frac{k_{j}}{N_{j n}}, \frac{k_{j}+1}{N_{j n}}\right],
$$

where $\boldsymbol{k}=\left(k_{1}, \ldots, k_{d}\right) \in \mathbb{Z}^{d}$ is a $d$-dimensional integer lattice point and $\left\{N_{j n}\right\}$ is an increasing and unbounded sequence of positive integers for each $j=1, \ldots, d$. Now, select the $n$-th data sites $\boldsymbol{x}_{\boldsymbol{k}}, \boldsymbol{k} \in \mathscr{D}_{n}$, by choosing an arbitrary point $\boldsymbol{x}_{k}$ from each $\Delta_{n}(\boldsymbol{k}) \cap T_{n}$ which is non-empty. Actually, each $\boldsymbol{x}_{\boldsymbol{k}}=\boldsymbol{x}_{k}^{(n)}$ depends on 
$n$ but to avoid complicated notation we often omit superscript $(n)$. We suppose that $\lim _{n \rightarrow \infty}\left|\mathscr{D}_{n}\right|=\infty$.

As the locations of the observations become more and more dense in an increasing sequence of domains, we call our setup infill-increasing (see [3, 12] for infill asymptotics).

Define the discrete parameter random field $Y_{n}(k)$ as follows. For arbitrary $n=$ $1,2, \ldots$ and $k \in D_{n}$, let $Y_{n}(\boldsymbol{k})$ be a Borel measurable function of $\varepsilon\left(\boldsymbol{x}_{k}^{(n)}\right)$.

We need the notion of $\alpha$-mixing (see, e. g., $[5,11]$ ). Let $\mathcal{A}$ and $\mathscr{B}$ be two $\sigma$ algebras in $\mathscr{F}$. The $\alpha$-mixing coefficient of $\mathcal{A}$ and $\mathscr{B}$ is

$$
\alpha(\mathcal{A}, \mathscr{B})=\sup \{|\mathbb{P}(A) \mathbb{P}(B)-\mathbb{P}(A B)|: A \in \mathcal{A}, B \in \mathscr{B}\} .
$$

The $\alpha$-mixing coefficient of $\left\{\varepsilon(\boldsymbol{x}): \boldsymbol{x} \in T_{\infty}\right\}$ is

$$
\alpha(r, u, v)=\sup \left\{\alpha\left(\mathcal{F}_{I_{1}}, \mathcal{F}_{I_{2}}\right): \varrho\left(I_{1}, I_{2}\right) \geq r,\left|I_{1}\right| \leq u,\left|I_{2}\right| \leq v\right\}
$$

where $I_{1}$ and $I_{2}$ are finite subsets in $T_{\infty}, \mathscr{F}_{I_{i}}=\sigma\left\{\varepsilon(\boldsymbol{x}): \boldsymbol{x} \in I_{i}\right\}, i=1,2$.

We say that the random field $\{\varepsilon(\boldsymbol{x})\}$ is $\alpha$-mixing if its mixing coefficients satisfy some conditions. All of these conditions mean a weak defence of the field, that is $\alpha(r, u, v)$ is small if $r$ is large.

We list the conditions that will be used in our theorems.

$$
\begin{gathered}
\int_{0}^{\infty} s^{d-1} \alpha^{\frac{\tau}{2+\tau}}(s, 1,1) d s<\infty \text { for some } 0<\tau<1 \\
\int_{0}^{\infty} s^{d-1} \alpha(s, i, j) d s<\infty \text { for } i+j \leq 4 \\
\alpha(s, 1, \infty)=\mathrm{o}\left(s^{-d}\right) \text { as } s \rightarrow \infty \\
\Lambda_{n}=\mathrm{O}\left(\lambda_{n}\right) \text { as } n \rightarrow \infty
\end{gathered}
$$

where

$$
\Lambda_{n}=\max _{1 \leq j \leq d} N_{j n}, \quad \lambda_{n}=\min _{1 \leq j \leq d} N_{j n} .
$$

Actually (2.3) means that $\lim _{n \rightarrow \infty} \alpha\left(s_{n}, 1, k_{n}\right) s_{n}^{d}=0$ if $s_{n} \rightarrow \infty$ and $k_{n} \rightarrow \infty$.

Let $\alpha_{n}(r, i, j)$ denote the $\alpha$-mixing coefficients of $Y_{n}(\boldsymbol{k})$. As $\boldsymbol{x}_{\boldsymbol{k}} \in \Delta_{n}(\boldsymbol{k})$ and $\boldsymbol{x}_{\boldsymbol{\ell}} \in \Delta_{n}(\boldsymbol{\ell})$ (where $\left.\boldsymbol{\ell}=\left(l_{1}, \ldots, l_{d}\right)\right)$, we have

$$
\varrho\left(\boldsymbol{x}_{\boldsymbol{k}}, \boldsymbol{x}_{\boldsymbol{\ell}}\right) \geq \max \left\{\frac{\left|k_{1}-l_{1}\right|-1}{N_{1 n}}, \ldots, \frac{\left|k_{d}-l_{d}\right|-1}{N_{d n}}\right\} \geq \frac{\varrho(\boldsymbol{k}, \boldsymbol{\ell})-1}{\Lambda_{n}}
$$

and

$$
\varrho\left(\boldsymbol{x}_{\boldsymbol{k}}, \boldsymbol{x}_{\boldsymbol{\ell}}\right) \leq \frac{\varrho(\boldsymbol{k}, \boldsymbol{\ell})+1}{\lambda_{n}}
$$

where $\Lambda_{n}, \lambda_{n}$ are given in (2.5). Therefore the $\alpha$-mixing coefficients $\alpha_{n}(r, i, j)$ of $Y_{n}(k)$ satisfy

$$
\alpha\left(\frac{r+1}{\lambda_{n}}, i, j\right) \leq \alpha_{n}(r, i, j) \leq \alpha\left(\frac{r-1}{\Lambda_{n}}, i, j\right), \quad r=1,2, \ldots
$$


Lemma 1. For $\gamma>0$ and positive integers $i, j, n$, the relation

$$
\sum_{r=1}^{\infty} r^{d-1} \alpha_{n}^{\gamma}(r, i, j) \leq c\left(1+\Lambda_{n}^{d} \int_{0}^{\infty} r^{d-1} \alpha^{\gamma}(r, i, j) d r\right)
$$

holds, where the constant $c$ depends only on $d$.

PROOF. We have

$$
\begin{aligned}
\sum_{r=1}^{\infty} r^{d-1} \alpha_{n}^{\gamma}(r, i, j) & \leq c\left(1+\sum_{r=1}^{\infty} r^{d-1} \alpha^{\gamma}\left(\frac{r}{\Lambda_{n}}, i, j\right)\right) \\
& \leq c\left(1+\sum_{r=2}^{\infty} \int_{r-1}^{r} s^{d-1} \alpha^{\gamma}\left(\frac{s}{\Lambda_{n}}, i, j\right) d s\right) \\
& \leq c\left(1+\int_{0}^{\infty} s^{d-1} \alpha^{\gamma}\left(\frac{s}{\Lambda_{n}}, i, j\right) d s\right) \\
& \leq c\left(1+\Lambda_{n}^{d} \int_{0}^{\infty} s^{d-1} \alpha^{\gamma}(s, i, j) d s\right),
\end{aligned}
$$

which leads us to (2.8).

Remark 1 (Davydov's inequality [5, p. 9]). The following well-known covariance inequalities are basic tools for mixing fields.

$$
|\operatorname{cov}(X, Y)| \leq 8[\alpha(\sigma(X), \sigma(Y))]^{\frac{1}{r}}\|X\|_{p}\|Y\|_{q}
$$

for $r, p, q \geq 1, r^{-1}+p^{-1}+q^{-1}=1$. In the special case where $X$ and $Y$ belong to $L_{\infty}$, we have

$$
|\operatorname{cov}(X, Y)| \leq 4[\alpha(\sigma(X), \sigma(Y))]\|X\|_{\infty}\|Y\|_{\infty} .
$$

The following inequality is a special case of the Rosenthal inequality. For the proof, see [5].

Lemma 2. Let $\xi_{\boldsymbol{k}}, k \in \mathbb{Z}^{d}$, be centered random variables with $\mathbb{E}\left|\xi_{\mathfrak{k}}\right|^{l+\tau}<\infty$, $k \in \mathbb{Z}^{d}$. Introduce the notation

$$
L(h, \tau, \mathscr{D})=\sum_{k \in \mathscr{D}}\left(\mathbb{E}\left|\xi_{k}\right|^{h+\tau}\right)^{\frac{h}{h+\tau}}
$$

if $1<h \leq 2, \tau \geq 0$ and $D$ is a finite set in $\mathbb{Z}^{d}$. Let

$$
c_{1,1}^{(\tau)}=1+\sum_{s=1}^{\infty} s^{d-1}\left[\alpha_{\xi}(s, 1,1)\right]^{\frac{\tau}{2+\tau}}
$$


where $\alpha_{\xi}(s, 1,1)$ is the $\alpha$-mixing coefficient of the field $\left\{\xi_{k}\right\}$. Now, let $1<l \leq 2$ and $\tau>0$. Assume that $c_{1,1}^{(\tau)}<\infty$. Then there is a constant c such that

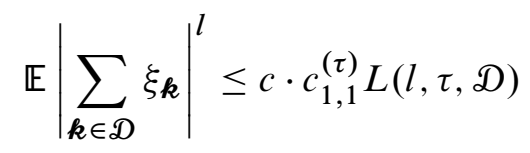

for any finite subset $\mathscr{D}$ of $\mathbb{Z}^{d}$.

We remark that the proof of the Rosenthal inequality (2.11) follows from (2.12) below, by using the so-called interpolation lemma. Details and the general form of the Rosenthal inequality can be found, e. g., in [8].

Remark 2. Using the notation of Lemma 2, let $\tau>0$, and assume that $c_{1,1}^{(\tau)}<\infty$. Then there is a constant $c$ such that

$$
\sum_{\boldsymbol{k}, \boldsymbol{\ell} \in \mathbb{D}}\left|\operatorname{cov}\left(\xi_{\boldsymbol{k}}, \xi_{\boldsymbol{\ell}}\right)\right| \leq c \cdot c_{1,1}^{(\tau)} L(2, \tau, \mathbb{D})
$$

for any finite subset $\mathscr{D}$ of $\mathbb{Z}^{d}$.

PROOF. For the sake of completeness we prove (2.12). By (2.9),

$$
\begin{aligned}
& \sum_{\boldsymbol{k}, \boldsymbol{\ell} \in \mathcal{D}}\left|\operatorname{cov}\left(\xi_{\boldsymbol{k}}, \xi_{\boldsymbol{\ell}}\right)\right| \leq \\
& \quad \leq \sum_{\boldsymbol{k} \in \mathcal{D}}\left\|\xi_{\boldsymbol{k}}\right\|_{2}^{2}+\sum_{\substack{\boldsymbol{k}, \boldsymbol{\ell} \in \mathcal{D} \\
\boldsymbol{k} \neq \boldsymbol{\ell}}} 8\left[\alpha_{\xi}(\|\boldsymbol{k}-\boldsymbol{\ell}\|, 1,1)\right]^{\frac{\tau}{2+\tau}}\left\|\xi_{\boldsymbol{k}}\right\|_{2+\tau}\left\|\xi_{\boldsymbol{\ell}}\right\|_{2+\tau}
\end{aligned}
$$

By the inequality between the geometric and arithmetic means, the above expression is majorized by

$$
\begin{aligned}
\sum_{\boldsymbol{k} \in \mathscr{D}}\left\|\xi_{\boldsymbol{k}}\right\|_{2+\tau}^{2}+ & \sum_{\substack{\boldsymbol{k}, \boldsymbol{\ell} \in \mathcal{D} \\
\boldsymbol{k} \neq \boldsymbol{\ell}}} 8\left[\alpha_{\xi}(\|\boldsymbol{k}-\boldsymbol{\ell}\|, 1,1)\right]^{\frac{\tau}{2+\tau}}\left\|\xi_{\boldsymbol{k}}\right\|_{2+\tau}^{2} \leq \\
& \leq \sum_{\boldsymbol{k} \in \mathcal{D}}\left\|\xi_{\boldsymbol{k}}\right\|_{2+\tau}^{2}+\sum_{\boldsymbol{k} \in \mathcal{D}} c \sum_{s=1}^{\infty} s^{d-1}\left[\alpha_{\xi}(s, 1,1)\right]^{\frac{\tau}{2+\tau}}\left\|\xi_{\boldsymbol{k}}\right\|_{2+\tau}^{2}
\end{aligned}
$$

This gives (2.12).

\section{MAIN THEOREMS}

Theorem 1. Let $\varepsilon(\boldsymbol{x})$ be a random field and let $Y_{n}(\boldsymbol{k})$ be a Borel measurable function of $\varepsilon\left(\boldsymbol{x}_{\boldsymbol{k}}^{(n)}\right), \boldsymbol{k} \in \mathscr{D}_{n}$. Suppose that $\mathbb{E} Y_{n}(\boldsymbol{k})=0$ and $\left|Y_{n}(\boldsymbol{k})\right|$ are uniformly 
bounded for $k \in \mathscr{D}_{n}, n=1,2, \ldots$ Let $S_{n}=\sum_{k \in D_{n}} Y_{n}(\boldsymbol{k}), n=1,2, \ldots$, $\sigma_{n}^{2}=\operatorname{var}\left(S_{n}\right)$. Let conditions (2.2), (2.3), and (2.4) be satisfied. Assume that

$$
\liminf _{n \rightarrow \infty} \frac{\sigma_{n}^{2}}{\Lambda_{n}^{d}\left|\mathscr{D}_{n}\right|}>0
$$

Then $\sigma_{n}^{-1} S_{n} \Rightarrow \mathcal{N}(0,1)$ as $n \rightarrow \infty$.

PROOF. We follow ideas of [11]. Throughout the proof we shall suppose that the random variables $Y_{n}(\boldsymbol{k})$ are uniformly bounded with the bound 1: $\left|Y_{n}(\boldsymbol{k})\right| \leq 1$, $k \in \mathscr{D}_{n}, n=1,2, \ldots$

Choose a sequence $\left\{m_{n}\right\}$ of positive integers such that $\lim _{n \rightarrow \infty} m_{n}=\infty$,

$$
\lim _{n \rightarrow \infty} \alpha\left(m_{n}, 1, \infty\right)\left|\mathscr{D}_{n}\right|^{\frac{1}{2}} \Lambda_{n}^{-\frac{d}{2}}=0
$$

and

$$
\lim _{n \rightarrow \infty} m_{n}^{-d}\left|\mathscr{D}_{n}\right|^{\frac{1}{2}} \Lambda_{n}^{-\frac{d}{2}}=\infty .
$$

To this end, let $x_{n}=\alpha(n, 1, \infty), y_{n}=n^{-d}$ and $z_{n}=\left|D_{n}\right|^{\frac{1}{2}} \Lambda_{n}^{-\frac{d}{2}}$ in Lemma 3 below. Then, by (2.3), $x_{n} / y_{n} \rightarrow 0$. Moreover $z_{n} \rightarrow \infty$ because $\left|\mathscr{D}_{n}\right| \Lambda_{n}^{-d} \geq c \mu\left(T_{n}\right) \rightarrow \infty$. Here, $\mu\left(T_{n}\right)$ is the Lebesgue measure of $T_{n}$.

Let $S_{n}(\boldsymbol{k})=\sum_{\boldsymbol{\ell} \in D_{n}, \varrho\left(\boldsymbol{x}_{\boldsymbol{k}}, \boldsymbol{x}_{\boldsymbol{\ell}}\right) \leq m_{n}} Y_{n}(\boldsymbol{\ell})$ and $S_{n}^{*}(\boldsymbol{k})=S_{n}-S_{n}(\boldsymbol{k})$ for any $\boldsymbol{k} \in$ $\mathscr{D}_{n}$. Let $a_{n}=\sum_{\boldsymbol{\ell} \in D_{n}} \mathbb{E}\left(Y_{n}(\boldsymbol{\ell}) S_{n}(\boldsymbol{\ell})\right), \bar{S}_{n}=a_{n}^{-\frac{1}{2}} S_{n}$, and $\bar{S}_{n}(\boldsymbol{k})=a_{n}^{-\frac{1}{2}} S_{n}(\boldsymbol{k})$. We have

$$
\sigma_{n}^{2}=\operatorname{var}\left(S_{n}\right)=a_{n}+\sum_{\boldsymbol{\ell} \in \mathscr{D}_{n}} \mathbb{E}\left(Y_{n}(\boldsymbol{\ell}) S_{n}^{*}(\boldsymbol{\ell})\right) .
$$

Using (2.10) and the same argument as in Lemma 1, we obtain

$$
\begin{aligned}
\sigma_{n}^{2}-a_{n} & =\left|\sum_{\boldsymbol{\ell} \in \mathscr{D}_{n}} \mathbb{E}\left(Y_{n}(\boldsymbol{\ell}) S_{n}^{*}(\boldsymbol{\ell})\right)\right| \\
& \leq \sum_{\boldsymbol{k}, \boldsymbol{\ell} \in \mathscr{D}_{n}, \varrho\left(\boldsymbol{x}_{\boldsymbol{k}}, \boldsymbol{x}_{\boldsymbol{\ell}}\right) \geq m_{n}}\left|\operatorname{cov}\left(Y_{n}(\boldsymbol{k}), Y_{n}(\boldsymbol{\ell})\right)\right| \\
& \leq c\left|\mathscr{D}_{n}\right| \sum_{s=m_{n} \lambda_{n}-1}^{\infty} s^{d-1} \alpha\left(\frac{s-1}{\Lambda_{n}}, 1,1\right) \\
& \leq c\left|\mathscr{D}_{n}\right| \int_{m_{n} \lambda_{n}-2}^{\infty} s^{d-1} \alpha\left(\frac{s-1}{\Lambda_{n}}, 1,1\right) d s \\
& \leq c\left|\mathscr{D}_{n}\right| \Lambda_{n}^{d} \int_{\frac{m_{n} \lambda_{n-3}}{\Lambda_{n}}}^{\infty} s^{d-1} \alpha(s, 1,1) d s \leq c\left|\mathscr{D}_{n}\right| \Lambda_{n}^{d}: \mathrm{o}(1) \leq \sigma_{n}^{2}: \mathrm{o}(1)
\end{aligned}
$$


In the last steps we used (2.2) and (3.1). Hence

$$
\lim _{n \rightarrow \infty} \frac{a_{n}}{\sigma_{n}^{2}}=1
$$

Therefore it is sufficient to prove the asymptotic normality of $\bar{S}_{n}$.

Since $\sup _{n} \mathbb{E} \bar{S}_{n}^{2}<\infty$, by Stein's lemma (see Remark 3 ) it is sufficient to prove that

$$
\lim _{n \rightarrow \infty} \mathbb{E}\left(\left(i t-\bar{S}_{n}\right) e^{i t \bar{S}_{n}}\right)=0 \text { for every } t \in \mathbb{R} .
$$

Consider the decomposition

$$
\left(i t-\bar{S}_{n}\right) e^{i t \bar{S}_{n}}=A_{1}-A_{2}-A_{3},
$$

where

$$
\begin{aligned}
& A_{1}=i t e^{i t \bar{S}_{n}}\left(1-\frac{1}{a_{n}} \sum_{\boldsymbol{\ell} \in D_{n}} Y_{n}(\boldsymbol{\ell}) S_{n}(\boldsymbol{\ell})\right), \\
& A_{2}=a_{n}^{-\frac{1}{2}} e^{i t \bar{S}_{n}} \sum_{\boldsymbol{\ell} \in D_{n}} Y_{n}(\boldsymbol{\ell})\left(1-i t \bar{S}_{n}(\boldsymbol{\ell})-e^{-i t \bar{S}_{n}(\boldsymbol{\ell})}\right), \\
& A_{3}=a_{n}^{-\frac{1}{2}} \sum_{\boldsymbol{\ell} \in D_{n}} Y_{n}(\boldsymbol{\ell}) e^{i t\left(\bar{S}_{n}-\bar{S}_{n}(\boldsymbol{\ell})\right)} .
\end{aligned}
$$

First we prove that $\lim _{n \rightarrow \infty} \mathbb{E}\left|A_{1}\right|^{2}=0$. We have

$$
\begin{aligned}
\mathbb{E}\left|A_{1}\right|^{2}=t^{2} a_{n}^{-2} \operatorname{var}\left(\sum_{\boldsymbol{\ell} \in D_{n}} Y_{n}(\boldsymbol{\ell}) S_{n}(\boldsymbol{\ell})\right)= \\
=t^{2} a_{n}^{-2} \sum_{\substack{\boldsymbol{j}, \boldsymbol{j}^{\prime}, \boldsymbol{\ell}, \boldsymbol{\ell}^{\prime} \in \mathbb{D}_{n}, \varrho\left(\boldsymbol{x}_{\boldsymbol{j}}, \boldsymbol{x}_{\boldsymbol{\ell}}\right) \leq m_{n}, \varrho\left(\boldsymbol{x}_{\boldsymbol{j}^{\prime}}, \boldsymbol{x}_{\boldsymbol{\ell}^{\prime}}\right) \leq m_{n}}} \operatorname{cov}\left(Y_{n}(\boldsymbol{j}) Y_{n}(\boldsymbol{\ell}), Y_{n}\left(\boldsymbol{j}^{\prime}\right) Y_{n}\left(\boldsymbol{\ell}^{\prime}\right)\right) .
\end{aligned}
$$

Now we distinguish two cases. First suppose that $\varrho\left(\boldsymbol{x}_{\boldsymbol{j}}, \boldsymbol{x}_{\boldsymbol{j}^{\prime}}\right)=k \geq \frac{3 m_{n} \Lambda_{n}}{\lambda_{n}}$. Then $\varrho\left(\left\{\boldsymbol{x}_{\boldsymbol{j}}, \boldsymbol{x}_{\boldsymbol{\ell}}\right\},\left\{\boldsymbol{x}_{\boldsymbol{j}^{\prime}}, \boldsymbol{x}_{\boldsymbol{\ell}^{\prime}}\right\}\right) \geq k-2 m_{n}$. Then, by the covariance inequality (2.10),

$$
\left|\operatorname{cov}\left(Y_{n}(\boldsymbol{j}) Y_{n}(\boldsymbol{\ell}), Y_{n}\left(\boldsymbol{j}^{\prime}\right) Y_{n}\left(\boldsymbol{\ell}^{\prime}\right)\right)\right| \leq 4 \alpha\left(k-2 m_{n}, 2,2\right),
$$

because $\left|Y_{n}(\boldsymbol{\ell})\right| \leq 1$ for each $\boldsymbol{\ell}$ and $n$. Now, we can choose $\boldsymbol{j}$ in $\left|\mathscr{D}_{n}\right|$ ways, then $\boldsymbol{\ell}$ at most in $m_{n}^{d} \Lambda_{n}^{d}$ ways, and when $\boldsymbol{j}^{\prime}$ is chosen, then we can choose $\boldsymbol{\ell}^{\prime}$ at most in 
$m_{n}^{d} \Lambda_{n}^{d}$ ways. So the expression in (3.6) is less than or equal to

$$
\begin{array}{r}
c t^{2} a_{n}^{-2}\left|D_{n}\right| m_{n}^{2 d} \Lambda_{n}^{2 d} \sup _{\boldsymbol{j} \in D_{n}} \sum_{\left\{\boldsymbol{j}^{\prime}: \varrho\left(\boldsymbol{x}_{\boldsymbol{j}}, \boldsymbol{x}_{\boldsymbol{j}^{\prime}}\right)=k \geq \frac{3 m_{n} \Lambda_{n}}{\lambda_{n}}\right\}} \alpha\left(\varrho\left(\boldsymbol{x}_{\boldsymbol{j}}, \boldsymbol{x}_{\boldsymbol{j}^{\prime}}\right)-2 m_{n}, 2,2\right) \\
\leq c t^{2} a_{n}^{-2}\left|\mathscr{D}_{n}\right| m_{n}^{2 d} \Lambda_{n}^{2 d} \sum_{s=3 m_{n} \Lambda_{n}-1}^{\infty} s^{d-1} \alpha\left(\frac{s-1}{\Lambda_{n}}-2 m_{n}, 2,2\right) .
\end{array}
$$

In the last step we used (2.6) and (2.7). We can majorize the above sum by the following integral:

$$
\begin{aligned}
c t^{2} a_{n}^{-2}\left|\mathscr{D}_{n}\right| m_{n}^{2 d} \Lambda_{n}^{2 d} \int_{3 m_{n} \Lambda_{n}-2}^{\infty} s^{d-1} \alpha\left(\frac{s-1}{\Lambda_{n}}-2 m_{n}, 2,2\right) d s \\
\leq c t^{2} a_{n}^{-2}\left|\mathscr{D}_{n}\right| m_{n}^{2 d} \Lambda_{n}^{2 d+1} \int_{m_{n}+\mathrm{o}(1)}^{\infty}\left(\Lambda_{n}\left(s+2 m_{n}\right)+1\right)^{d-1} \alpha(s, 2,2) d s \\
\leq c t^{2} a_{n}^{-2}\left|\mathscr{D}_{n}\right| m_{n}^{2 d} \Lambda_{n}^{3 d} \int_{0}^{\infty} s^{d-1} \alpha(s, 2,2) d s .
\end{aligned}
$$

Now, we can use (3.1) and (3.4) to show that $a_{n}^{-1} \leq c\left|\mathscr{D}_{n}\right|^{-1} \Lambda_{n}^{-d}$. Therefore the above expression is majorized by $c\left|\mathscr{D}_{n}\right|^{-1} m_{n}^{2 d} \Lambda_{n}^{d}$, which converges to 0 , as $n \rightarrow \infty$, by the choice of $m_{n}$ (see (3.2), (3.3)).

In the second case we suppose that $\varrho\left(\boldsymbol{x}_{\boldsymbol{j}}, \boldsymbol{x}_{\boldsymbol{j}^{\prime}}\right)=k<\frac{3 m_{n} \Lambda_{n}}{\lambda_{n}}$. Let

$$
h=\inf \left\{\varrho\left(\boldsymbol{x}_{\boldsymbol{j}}, \boldsymbol{x}_{\boldsymbol{j}^{\prime}}\right), \varrho\left(\boldsymbol{x}_{\boldsymbol{j}}, \boldsymbol{x}_{\boldsymbol{\ell}}\right), \varrho\left(\boldsymbol{x}_{\boldsymbol{j}}, \boldsymbol{x}_{\boldsymbol{\ell}^{\prime}}\right)\right\}
$$

Then, by the covariance inequality (2.10),

$$
\begin{aligned}
\left|\operatorname{cov}\left(Y_{n}(\boldsymbol{j}) Y_{n}(\boldsymbol{\ell}), Y_{n}\left(\boldsymbol{j}^{\prime}\right) Y_{n}\left(\boldsymbol{\ell}^{\prime}\right)\right)\right| & \\
\leq\left|\mathbb{E}\left(Y_{n}(\boldsymbol{j}) Y_{n}(\boldsymbol{\ell}) Y_{n}\left(\boldsymbol{j}^{\prime}\right) Y_{n}\left(\boldsymbol{\ell}^{\prime}\right)\right)\right| & +\left|\mathbb{E}\left(Y_{n}(\boldsymbol{j}) Y_{n}(\boldsymbol{\ell})\right)\right|\left|\mathbb{E}\left(Y_{n}\left(\boldsymbol{j}^{\prime}\right) Y_{n}\left(\boldsymbol{\ell}^{\prime}\right)\right)\right| \\
& \leq 4 \alpha(h, 1,3)+4 \alpha(h, 1,1) \leq 8 \alpha(h, 1,3),
\end{aligned}
$$

because $\left|Y_{n}(\boldsymbol{\ell})\right| \leq 1$ for each $\boldsymbol{\ell}$ and $n$. Suppose that $h=\varrho\left(\boldsymbol{x}_{\boldsymbol{j}}, \boldsymbol{x}_{\boldsymbol{\ell}}\right)$ (the other two cases can be studied similarly). Then we can choose $j$ in $\left|\mathscr{D}_{n}\right|$ ways, $j^{\prime}$ at most in $\Lambda_{n}^{d}\left(3 m_{n} \Lambda_{n}\left(\lambda_{n}\right)^{-1}\right)^{d}$ ways, and $\ell^{\prime}$ at most in $\Lambda_{n}^{d} m_{n}^{d}$ ways. So the expression in 
(3.6) is less than or equal to

$$
\begin{aligned}
& c t^{2} a_{n}^{-2} \mid D_{n} \mid \Lambda_{n}^{d}\left(\frac{3 m_{n} \Lambda_{n}}{\lambda_{n}}\right)^{d} \Lambda_{n}^{d} m_{n}^{d} \sum_{\left\{\boldsymbol{\ell}: \varrho\left(\boldsymbol{x}_{\boldsymbol{j}}, \boldsymbol{x}_{\boldsymbol{\ell}}\right)=h \leq m_{n}\right\}} \alpha(h, 1,3) \\
& \leq c t^{2} a_{n}^{-2}\left|\mathscr{D}_{n}\right| \Lambda_{n}^{3 d} m_{n}^{2 d} \lambda_{n}^{-d} \sum_{\left\{\boldsymbol{\ell}: \varrho(\boldsymbol{j}, \boldsymbol{\ell})=k \leq \Lambda_{n} m_{n}+1\right\}} \alpha\left(\frac{k-1}{\Lambda_{n}}, 1,3\right) \\
& \leq c t^{2} a_{n}^{-2}\left|\mathscr{D}_{n}\right| \Lambda_{n}^{2 d} m_{n}^{2 d}\left(1+\sum_{k=1}^{\Lambda_{n} m_{n}+1} k^{d-1} \alpha\left(\frac{k-1}{\Lambda_{n}}, 1,3\right)\right) \\
& \leq c t^{2} a_{n}^{-2}\left|\mathscr{D}_{n}\right| \Lambda_{n}^{2 d} m_{n}^{2 d}\left(1+\int_{0}^{\Lambda_{n} m_{n}+1} s^{d-1} \alpha\left(\frac{s-1}{\Lambda_{n}}, 1,3\right) d s\right) \\
& \leq c t^{2} a_{n}^{-2}\left|D_{n}\right| \Lambda_{n}^{3 d} m_{n}^{2 d} \rightarrow 0, n \rightarrow \infty,
\end{aligned}
$$

as it was shown above. Therefore both parts of the sum in (3.6) converge to 0 , so $\lim _{n \rightarrow \infty} \mathbb{E}\left|A_{1}\right|^{2}=0$.

Now, we turn to $A_{2}$. By Taylor's expansion, we have

$$
\left|1-i t \bar{S}_{n}(\boldsymbol{\ell})-e^{-i t \bar{S}_{n}(\boldsymbol{\ell})}\right| \leq c t^{2} \bar{S}_{n}^{2}(\boldsymbol{\ell})
$$

and, therefore,

$$
\begin{aligned}
\mathbb{E}\left|A_{2}\right| & \leq a_{n}^{-\frac{1}{2}} \sum_{\boldsymbol{\ell} \in \mathscr{D}_{n}} \mathbb{E}\left|1-i t \bar{S}_{n}(\boldsymbol{\ell})-e^{-i t \bar{S}_{n}(\boldsymbol{\ell})}\right| \\
& \leq a_{n}^{-\frac{1}{2}}\left|\mathscr{D}_{n}\right| \sup _{\boldsymbol{\ell} \in D_{n}} \mathbb{E}\left(c t^{2} \bar{S}_{n}^{2}(\boldsymbol{\ell})\right) \\
& \leq c a_{n}^{-\frac{3}{2}}\left|\mathscr{D}_{n}\right| \sup _{\boldsymbol{\ell}} \sum_{\varrho(\boldsymbol{\ell}: ; \boldsymbol{j}) \leq \Lambda_{n} m_{n}, \varrho\left(\boldsymbol{\ell}: \dot{\boldsymbol{j}}^{\prime}\right) \leq \Lambda_{n} m_{n}}\left|\operatorname{cov}\left(Y_{n}(\boldsymbol{j}), Y_{n}\left(\boldsymbol{j}^{\prime}\right)\right)\right| \\
& \leq c a_{n}^{-\frac{3}{2}}\left|\mathscr{D}_{n}\right| m_{n}^{d} \Lambda_{n}^{2 d} \rightarrow 0
\end{aligned}
$$

as $n \rightarrow \infty$, because of the choice of $m_{n}$, the relation between $a_{n}$ and $\sigma_{n}^{2},(3.1)$ and (3.2). In the above calculation, we have applied the covariance inequality and the majorization of the sum by an integral as we did before. Hence, $\lim _{n \rightarrow \infty} \mathbb{E}\left|A_{2}\right|=0$.

Now, we shall prove that $\lim _{n \rightarrow \infty} \mathbb{E} A_{3}=0$.

$$
\begin{aligned}
\left|\mathbb{E} A_{3}\right| \leq a_{n}^{-\frac{1}{2}} \sum_{\boldsymbol{\ell} \in \mathscr{D}_{n}}\left|\operatorname{cov}\left(Y_{n}(\boldsymbol{\ell}), e^{i t\left(\bar{S}_{n}-\bar{S}_{n}(\boldsymbol{\ell})\right)}\right)\right| \leq \\
\quad \leq a_{n}^{-\frac{1}{2}}\left|\mathscr{D}_{n}\right| \alpha\left(m_{n}, 1, \infty\right) \leq c\left|\Lambda_{n}\right|^{-\frac{d}{2}}\left|\mathscr{D}_{n}\right|^{\frac{1}{2}} \alpha\left(m_{n}, 1, \infty\right) \rightarrow 0,
\end{aligned}
$$

by the choice of $m_{n}$. 
So (3.5) is proved and, therefore, the theorem is proved.

Theorem 2. Let $\varepsilon(\boldsymbol{x})$ be a random field and let $Y_{n}(\boldsymbol{k})$ be a Borel measurable function of $\varepsilon\left(\boldsymbol{x}_{k}^{(n)}\right), \boldsymbol{k} \in \mathbb{D}_{n}$. Suppose that $\mathbb{E} Y_{n}(\boldsymbol{k})=0$ for $\boldsymbol{k} \in \mathscr{D}_{n}, n=1,2, \ldots$. Let $S_{n}=\sum_{k \in D_{n}} Y_{n}(\boldsymbol{k}), n=1,2, \ldots, \sigma_{n}^{2}=\operatorname{var}\left(S_{n}\right)$. Suppose that there exists $a$ $\tau>0$ such that (2.1)is satisfied and

$$
\left\{\left|Y_{n}(k)\right|^{2+\tau}: k \in \mathscr{D}_{n}, n=1,2, \ldots\right\} \text { are uniformly integrable. }
$$

Then

$$
\limsup _{n \rightarrow \infty} \frac{1}{\Lambda_{n}^{d}\left|\mathscr{D}_{n}\right|} \sum_{\boldsymbol{k}, \boldsymbol{\ell} \in \mathscr{D}_{n}}\left|\operatorname{cov}\left(Y_{n}(\boldsymbol{k}), Y_{n}(\boldsymbol{\ell})\right)\right|<\infty .
$$

If, additionally, conditions (2.2), (2.3), (2.4), and (3.1) are satisfied, then $\sigma_{n}^{-1} S_{n} \Rightarrow$ $\mathcal{N}(0,1)$ as $n \rightarrow \infty$.

ProOF. First we prove (3.8). By the Rosenthal inequality (2.11),

$$
\begin{aligned}
& \sum_{\boldsymbol{k}, \boldsymbol{\ell} \in \mathscr{D}_{n}}\left|\operatorname{cov}\left(Y_{n}(\boldsymbol{k}), Y_{n}(\boldsymbol{\ell})\right)\right| \leq \\
& \leq c\left(1+\sum_{s=1}^{\infty}\left[\alpha_{n}(s, 1,1)\right]^{\frac{\tau}{2+\tau}} s^{d-1}\right) \sum_{\boldsymbol{k} \in \mathcal{D}}\left(\mathbb{E}\left|Y_{\boldsymbol{k}}\right|^{2+\tau}\right)^{\frac{2}{2+\tau}} .
\end{aligned}
$$

Due to Lemma 1, this expression is majorized by

$$
\begin{aligned}
c \cdot\left(1+\Lambda_{n}^{d} \int_{0}^{\infty} s^{d-1} \alpha^{\frac{\tau}{2+\tau}}(s, 1,1) d s\right) \times & \\
& \times\left|\mathscr{D}_{n}\right| \sup \left\{\left\|Y_{n}(k)\right\|_{2+\tau}^{2}: k \in \mathscr{D}_{n}, n=1,2, \ldots\right\} .
\end{aligned}
$$

Therefore, (3.7) and (2.1) imply (3.8).

Now, we show that it is sufficient to prove the theorem for uniformly bounded random variables $\left\{Y_{n}(k): k \in \mathscr{D}_{n}, n=1,2, \ldots\right\}$. So Theorem 1 will imply the result. We follow the ideas of Ibragimov and Linnik [6]. Let $L>0$ and define the truncated variables by the superscript $(L)$ and the remainder by breve and superscript $(L): X^{(L)}=X \cdot \mathrm{I}\{X \in[-L, L]\}, \breve{X}^{(L)}=X-X^{(L)}$. Let $Z_{n}=S_{n} / \sigma_{n}$ be the standardized sum,

$$
Z_{n}^{(L)}=\frac{1}{\sigma_{n}} \sum_{k \in D_{n}}\left(Y_{n}^{(L)}(k)-\mathbb{E} Y_{n}^{(L)}(k)\right)
$$

be the normalized sum of the truncated variables, and

$$
\breve{Z}_{n}^{(L)}=\frac{1}{\sigma_{n}} \sum_{k \in D_{n}}\left(\breve{Y}_{n}^{(L)}(k)-\mathbb{E} \breve{Y}_{n}^{(L)}(k)\right)
$$

be the normalized sum of the remainders. Then $Z_{n}=Z_{n}^{(L)}+\breve{Z}_{n}^{(L)}$ and $\mathbb{E} Z_{n}^{2}=1$. 
By the Rosenthal inequality, (2.8), and (2.1),

$$
\begin{aligned}
\mathbb{E}\left(\breve{Z}_{n}^{(L)}\right)^{2}=\mathbb{E} \mid \frac{1}{\sigma_{n}} \sum_{k \in \mathscr{D}_{n}}\left(\breve{Y}_{n}^{(L)}(\boldsymbol{k})\right. & \left.-\mathbb{E} \breve{Y}_{n}^{(L)}(\boldsymbol{k})\right)\left.\right|^{2} \leq \\
& \leq c \frac{\Lambda_{n}^{d}\left|\mathscr{D}_{n}\right|}{\sigma_{n}^{2}} \sup _{k \in \mathscr{D}_{n}}\left\|\breve{Y}_{n}^{(L)}(\boldsymbol{k})\right\|_{2+\tau}^{2} \rightarrow 0
\end{aligned}
$$

as $L \rightarrow \infty$. We remark that this convergence is uniform in $n$. In the last step we used (3.1) and (3.7).

Let $\sigma_{n}^{2}(L)=\operatorname{var}\left(\sum_{k \in D_{n}} Y_{n}^{(L)}(k)\right)$ be the variance of the sum of the truncated variables. Now

$$
\begin{aligned}
& \frac{\sigma_{n}^{2}(L)}{\sigma_{n}^{2}}-1=\mathbb{E}\left(Z_{n}^{(L)}\right)^{2}-\mathbb{E}\left(Z_{n}\right)^{2}= \\
& \quad=\mathbb{E}\left(Z_{n}-\breve{Z}_{n}^{(L)}\right)^{2}-\mathbb{E}\left(Z_{n}\right)^{2}=\mathbb{E}\left(\breve{Z}_{n}^{(L)}\right)^{2}-2 \mathbb{E}\left(Z_{n} \breve{Z}_{n}^{(L)}\right) .
\end{aligned}
$$

By (3.9), and using the Cauchy inequality for the second term, the above expression converges to 0 , as $L \rightarrow \infty$, uniformly in $n$. Therefore,

$$
\lim _{L \rightarrow \infty} \sup _{n \geq 1}\left|\frac{\sigma_{n}^{2}(L)}{\sigma_{n}^{2}}-1\right|=0 .
$$

Now,

$$
\begin{aligned}
& \left|\mathbb{E} e^{i t Z_{n}}-e^{-t^{2} / 2}\right| \leq \\
& \leq \mathbb{E}\left|e^{i t \breve{Z}_{n}^{(L)}}-1\right|+\left|\mathbb{E} e^{i t Z_{n}^{(L)}}-e^{-\frac{\sigma_{n}^{2}(L)}{\sigma_{n}^{2}} \frac{t^{2}}{2}}\right|+\left|e^{-\frac{\sigma_{n}^{2}(L)}{\sigma_{n}^{2}} \frac{t^{2}}{2}}-e^{-\frac{t^{2}}{2}}\right| \leq \\
& \quad \leq|t| \mathbb{E}\left|\breve{Z}_{n}^{(L)}\right|+\sup _{v \in\left[1-\delta_{L}, 1+\delta_{L}\right]}\left|\mathbb{E} e^{i t v U_{n}}-e^{-\frac{(t v)^{2}}{2}}\right|+\frac{t^{2}}{2} \delta_{L}
\end{aligned}
$$

where $\delta_{L}=\sup _{n \geq 1}\left|\frac{\sigma_{n}^{2}(L)}{\sigma_{n}^{2}}-1\right|$ and $U_{n}=\frac{1}{\sigma_{n}(L)} \sum_{k \in D_{n}}\left(Y_{n}^{(L)}(k)-\mathbb{E} Y_{n}^{(L)}(k)\right)$.

By (3.10), $\lim _{L \rightarrow \infty} \delta_{L}=0$. If the theorem is valid for bounded random variables, then $U_{n}$ is asymptotically standard normal, therefore (3.11) implies that

$$
\limsup _{n \rightarrow \infty}\left|\mathbb{E} e^{i t Z_{n}}-e^{-t^{2} / 2}\right| \leq|t| \sqrt{\sup _{n \geq 1} \mathbb{E}\left(\breve{Z}_{n}^{(L)}\right)^{2}}+\frac{t^{2}}{2} \delta_{L} .
$$

However, using (3.9), the last expression converges to 0 , as $L \rightarrow \infty$. Therefore, the theorem is valid.

In the proof of Theorem 1 we used the existence of the following subsequence $m_{n}$. For the sake of completeness we give a proof of the existence. 
Lemma 3. Let $x_{n} \downarrow 0, y_{n} \downarrow 0, z_{n} \rightarrow \infty$ be real sequences such that $y_{n}=n^{-d}$ with $d>0$ and $x_{n} / y_{n} \rightarrow 0$. Then there exists a sequence $m_{n}$ of positive integers such that $m_{n} \rightarrow \infty, x_{m_{n}} z_{n} \rightarrow 0$, and $y_{m_{n}} z_{n} \rightarrow \infty$.

PROOF. As $\tau_{n}=x_{n} / y_{n} \rightarrow 0$ we can find an increasing unbounded sequence $u_{k}$ of positive numbers such that $\tau_{n} \leq k^{-2}$ if $n \geq u_{k}^{1 / d}$. As $z_{n} \rightarrow \infty$, we can find an increasing unbounded sequence $\{n(k)\}$ of positive integers such that $z_{n} \geq k u_{k}$ if $n \geq n(k)$. Now let $\alpha_{m}=1 / k$ if $n(k) \leq m<n(k+1)$ for each positive integer $k$. Therefore, $\alpha_{m} \rightarrow 0$ and for each positive integer $k$ we have $z_{m} \alpha_{m} \geq k u_{k} / k=u_{k}$ if $n(k) \leq m<n(k+1)$.

Now let $m_{n}=\left[\left(z_{n} \alpha_{n}\right)^{1 / d}\right]+1$ for each $n$, where [.] denotes the integer part of a number. It is easy to see that $m_{n} \uparrow \infty$. Using the above considerations, for each positive integer $k$ we have

$$
\frac{\tau_{m_{n}}}{\alpha_{n}} \leq \frac{k^{-2}}{k^{-1}}=k^{-1} \text { if } n(k) \leq n<n(k+1) .
$$

Therefore, by the definition of $\tau_{n}$, the definition of $m_{n}$ and (3.12), we get

$$
x_{m_{n}} z_{n}=\tau_{m_{n}} y_{m_{n}} z_{n} \leq \tau_{m_{n}}\left(z_{n} \alpha_{n}\right)^{-1} z_{n}=\tau_{m_{n}} \alpha_{n}^{-1} \rightarrow 0
$$

if $n \rightarrow \infty$. Moreover, by the definition of $\alpha_{n}$, we obtain

$$
y_{m_{n}} z_{n}=\frac{z_{n}}{m_{n}^{d}}=\frac{z_{n}}{z_{n} \alpha_{n}}\left\{\frac{\left(z_{n} \alpha_{n}\right)^{1 / d}}{\left[\left(z_{n} \alpha_{n}\right)^{1 / d}\right]+1}\right\}^{d} \rightarrow \infty
$$

as $n \rightarrow \infty$.

Remark 3 (Stein's lemma $[11,14]$ ). Let $\left\{v_{n}\right\}$ be a sequence of probabilities on $\mathbb{R}$ such that $\sup _{n} \int_{\mathbb{R}} x^{2} v_{n}(d x)<\infty$ and

$$
\lim _{n \rightarrow \infty} \int_{\mathbb{R}}(i t-x) e^{i t x} v_{n}(d x)=0
$$

for every $t \in \mathbb{R}$. Then $v_{n} \Rightarrow \mathcal{N}(0,1)$ as $n \rightarrow \infty$.

\section{EXTENSIONS OF MAIN THEOREMS}

Corollary 1. In Theorems 1 and 2, instead of (3.1), assume that

$$
\lim _{n \rightarrow \infty} \Lambda_{n}^{-d}\left|\mathscr{D}_{n}\right|^{-1} \sigma_{n}^{2}=\sigma^{2} \text {. }
$$

Then $\left(\Lambda_{n}^{d}\left|\mathscr{D}_{n}\right|\right)^{-\frac{1}{2}} S_{n} \Rightarrow \mathcal{N}\left(0, \sigma^{2}\right)$ as $n \rightarrow \infty$.

ProOF. First assume that $\sigma^{2}>0$. Then (3.1) is satisfied and therefore $\sigma_{n}^{-1} S_{n} \Rightarrow$ $\mathcal{N}(0,1)$. So

$$
\left(\Lambda_{n}^{d}\left|\mathscr{D}_{n}\right|\right)^{-\frac{1}{2}} S_{n}=\left(\frac{\sigma_{n}^{2}}{\Lambda_{n}^{d}\left|\mathscr{D}_{n}\right|}\right)^{\frac{1}{2}} \sigma_{n}^{-1} S_{n} \Rightarrow \sigma \mathcal{N}(0,1)=\mathcal{N}\left(0, \sigma^{2}\right)
$$


If $\sigma^{2}=0$, then $\operatorname{var}\left[\left(\Lambda_{n}^{d}\left|\mathscr{D}_{n}\right|\right)^{-\frac{1}{2}} S_{n}\right]=\left(\Lambda_{n}^{d}\left|\mathscr{D}_{n}\right|\right)^{-1} \sigma_{n}^{2} \rightarrow 0$ as $n \rightarrow \infty$. Therefore, $\left(\Lambda_{n}^{d}\left|D_{n}\right|\right)^{-\frac{1}{2}} S_{n}$ converges to 0 in $L^{2}$, so it converges to the degenerate normal law $\mathcal{N}(0,0)$ in distribution.

Now we turn to $p$-dimensional extensions of Theorems 1 and 2. Our Theorem 3 is the same as Theorem 3.1 of [10].

Theorem 3. Let $\varepsilon(\boldsymbol{x})$ be a random field and let the $p$-dimensional random vector $Y_{n}(\boldsymbol{k})$ be a Borel measurable function of $\varepsilon\left(\boldsymbol{x}_{\boldsymbol{k}}^{(n)}\right), \boldsymbol{k} \in \mathbb{D}_{n}$. Suppose that $\mathbb{E} Y_{n}(\boldsymbol{k})=$ 0 and $\left\|Y_{n}(\boldsymbol{k})\right\|$ are uniformly bounded for $k \in \mathscr{D}_{n}, n=1,2, \ldots$ Let $S_{n}=$ $\sum_{k \in \mathcal{D}_{n}} Y_{n}(\boldsymbol{k}), n=1,2, \ldots, \Sigma_{n}=\operatorname{var}\left(S_{n}\right)$. Suppose that conditions (2.2), (2.3), and (2.4) are satisfied. Assume that the limit

$$
\lim _{n \rightarrow \infty}\left(\Lambda_{n}^{-d}\left|\mathscr{D}_{n}\right|^{-1} \Sigma_{n}\right)=\Sigma
$$

exists. Then $\left(\Lambda_{n}^{d}\left|D_{n}\right|\right)^{-\frac{1}{2}} S_{n} \Rightarrow \mathcal{N}(0, \Sigma)$ as $n \rightarrow \infty$.

PROOF. Consider the one dimensional random field $\left\{a^{\top} Y_{n}(\boldsymbol{k})\right\}$, where $a \in \mathbb{R}^{p}$ is arbitrary $\left(a^{\top}\right.$ is the transpose of $a$ ). Apply Theorem 1 and Corollary 1 to the field $\left\{a^{\top} Y_{n}(k)\right\}$.

Our Theorem 4 is the same as Remark 4.3 in [7].

Theorem 4. Let $\varepsilon(\boldsymbol{x})$ be a random field. For each $n=1,2, \ldots$, and for each $k \in D_{n}$, let $Y_{n}(\boldsymbol{k})$ be a centered $p$-dimensional random vector that is $\varepsilon\left(\boldsymbol{x}_{k}^{(n)}\right)$ measurable. Let $S_{n}=\sum_{k \in D_{n}} Y_{n}(k), n=1,2, \ldots, \Sigma_{n}=\operatorname{var}\left(S_{n}\right)$. Assume that conditions (2.2), (2.3), and (2.4) are satisfied. Moreover, assume that there exists a $\tau>0$ such that $(2.1)$ is satisfied, and

$$
\left\{\left\|Y_{n}(k)\right\|^{2+\tau}: k \in \mathscr{D}_{n}, n=1,2, \ldots\right\} \text { are uniformly integrable. }
$$

Assume that

$$
\liminf _{n \rightarrow \infty} \lambda_{\min }\left(\Lambda_{n}^{-d}\left|\mathscr{D}_{n}\right|^{-1} \Sigma_{n}\right)>0 .
$$

Then $\Sigma_{n}^{-\frac{1}{2}} S_{n} \Rightarrow \mathcal{N}\left(0, I_{p}\right)$ as $n \rightarrow \infty$.

Here, $\lambda_{\min }(A)$ denotes the minimal eigenvalue of the matrix $A$ and $I_{p}$ is the $p \times p$ type unit matrix.

Proof. Apply Theorem 2 to the field $\left\{a^{\top} Y_{n}(k)\right\}$.

Several other versions of the above theorems can be obtained. For example, the uniform integrability condition can be substituted by a strong stationarity condition and an integrability condition. 


\section{REFERENCES}

[1] Bolthausen, E.: On the central limit theorem for stationary mixing random fields. Ann. Probability, 10 (1982), 1047-1050.

[2] BRADLEY, C.: Basic properties of strong mixing condition. A survey and some open questions. Probability Surveys, 2 (2005), 107-144.

[3] Cressie, N. A. C.: Statistics for Spatial Data. Wiley, New York, 1991.

[4] Dedecker, J.: A central limit theorem for stationary random fields, Probab. Theory Relat. Fields., 110 (1998), 397-426.

[5] Doukhan, P.: Mixing. Properties and Examples. Lecture Notes in Statistics 85, Springer, New York. (1994).

[6] Ibragimov, I. A. And Linnik, Yu. V. : Independent and Stationary Sequences of Random Variables, Wolters-Noordhoff, Groningen. (1971).

[7] FAZEKAS, I. AND KuKush, A. G.: Infill asymptotics inside increasing domains for the least squares estimator in linear models. Stat. Inf. Stoch. Proc., 3 (2000), 199-223.

[8] Fazekas, I., Kunush, A. G. And TómÁcs, T.: On the Rosenthal inequality for mixing fields, Ukrainian Math. J., 52 (2000), No. 2, 266-276.

[9] Fazekas, I. AND Kukush, A. G.: A central limit theorem for mixing random fields and its statistical applications. In: Limit theorems in probability and statistics (Balatonlelle, 1999), Vol. II, pp. 59-75, János Bolyai Math. Soc., Budapest (2002).

[10] FAZEKAS, I.: Limit theorems for the empirical distribution function in the spatial case. Statistics \& Probability Letters, 62 (2003), 251-262.

[11] Guyon, X.: Random Fields on a Network. Modeling, Statistics, and Applications, Springer, New York, 1995.

[12] LAHIRI, S. N.: On inconsistency of estimators based on spatial data under infill asymptotics, Sankhya, 58 (1996), Ser. A, 403-417.

[13] Merlevède, F., Peligrad, M. ANd Utev, S.: Recent advances in invariance principles for stationary sequences, Probability Surveys, 3 (2006), 1-36.

[14] STEIN, C.: A bound for the error in the normal approximation to the distribution of a sum of dependent random variables. Proc. of the Sixth Berkeley Sympos. on Math. Stat. Prob., 2 (1972), 583-602.

Author's address

Zsolt Karácsony:

Institute of MAThematics, University of MiskolC, H-3515 MiskolC-EgYetemVÁRos, HUNGARY

E-mail address: matkzs@uni-miskolc.hu 